\title{
Interleukin-1 primes human mesenchymal stem cells towards an anti-inflammatory and pro-trophic phenotype in vitro
}

Elena Redondo-Castro ${ }^{1}$, Catriona Cunningham', Jonjo Miller ${ }^{1}$, Licia Martuscelli ${ }^{1}$, Sarah Aoulad-Ali ${ }^{1}$, Nancy J. Rothwell ${ }^{1}$, Cay M. Kielty ${ }^{1,2}$, Stuart M. Allan ${ }^{1}$ and Emmanuel Pinteaux ${ }^{1 *}$

\begin{abstract}
Background: Inflammation is a key contributor to central nervous system (CNS) injury such as stroke, and is a major target for therapeutic intervention. Effective treatments for CNS injuries are limited and applicable to only a minority of patients. Stem cell-based therapies are increasingly considered for the treatment of CNS disease, because they can be used as in-situ regulators of inflammation, and improve tissue repair and recovery. One promising option is the use of bone marrow-derived mesenchymal stem cells (MSCs), which can secrete antiinflammatory and trophic factors, can migrate towards inflamed and injured sites or can be implanted locally. Here we tested the hypothesis that pre-treatment with inflammatory cytokines can prime MSCs towards an antiinflammatory and pro-trophic phenotype in vitro.

Methods: Human MSCs from three different donors were cultured in vitro and treated with inflammatory mediators as follows: interleukin (IL)-1a, IL-1 $\beta$, tumour necrosis factor alpha (TNF-a) or interferon- $\gamma$. After $24 \mathrm{~h}$ of treatment, cell supernatants were analysed by ELISA for expression of granulocyte-colony stimulating factor (G-CSF), IL-10, brain-derived neurotrophic factor (BDNF), nerve growth factor (NGF), IL-1 receptor antagonist (IL-1Ra) and vascular endothelial growth factor (VEGF). To confirm the anti-inflammatory potential of MSCs, immortalised mouse microglial BV2 cells were treated with bacterial lipopolysaccharide (LPS) and exposed to conditioned media (CM) of naïve or IL-1-primed MSCS, and levels of secreted microglial-derived inflammatory mediators including TNF-a, IL-10, G-CSF and IL-6 were measured by ELISA.
\end{abstract}

Results: Unstimulated MSCs constitutively expressed anti-inflammatory cytokines and trophic factors (IL-10, VEGF, BDNF, G-CSF, NGF and IL-1Ra). MSCs primed with IL-1a or IL-1 13 showed increased secretion of G-CSF, which was blocked by IL-1Ra. Furthermore, LPS-treated BV2 cells secreted less inflammatory and apoptotic markers, and showed increased secretion of the anti-inflammatory IL-10 in response to treatment with CM of IL-1-primed MSCs compared with CM of unprimed MSCS.

Conclusions: Our results demonstrate that priming MSCs with IL-1 increases expression of trophic factor G-CSF through an IL-1 receptor type 1 (IL-1R1) mechanism, and induces a reduction in the secretion of inflammatory mediators in LPS-activated microglial cells. The results therefore support the potential use of preconditioning treatments of stem cells in future therapies.

Keywords: Stroke, Human mesenchymal stem cells, Cytokines, Priming, Bone marrow-derived stromal cells, Interleukin-1

\footnotetext{
* Correspondence: emmanuel.pinteaux@manchester.ac.uk

${ }^{1}$ Faculty of Biology, Medicine and Health, University of Manchester,

Manchester, UK

Full list of author information is available at the end of the article
} 


\section{Background}

Stem cells are undifferentiated cells found in many adult tissues, the function of which is renewal of damaged tissues during ageing or after disease and injuries. Because of their regenerative properties, these cells are being increasingly considered as new therapeutic agents for the treatment of central nervous system (CNS) diseases. Mesenchymal stem (or stromal) cells (MSCs) are one type of multipotent stem cells that can be isolated and cultured from several tissues [1,2] and differentiated into several cell lineages $[3,4]$. MSCs sense signals from tumours [5] and injured, inflamed or ischemic tissues [6], migrate towards those sites and are even able to cross the blood-brain barrier [7]. Once infiltrated into the brain, MSCs produce an array of mediators such as cytokines and growth factors $[8,9]$ that promote tissue repair mainly by activating endogenous repair mechanisms [10, 11], and by acting as temporal immunesuppressants $[1,6]$. These properties make MSCs ideal candidates for cell-based therapies, particularly for the treatment of CNS disorders such as stroke, Huntington's disease, amyotrophic lateral sclerosis or Parkinson's disease [12]. The safety and efficacy of MSCs have already been demonstrated mostly in pre-clinical models of ischaemic stroke $[2,9,13]$, intracerebral haemorrhage [14], amyotrophic lateral sclerosis [15] and Alzheimer's disease [16]. However, the precise mechanisms by which MSCs exert beneficial effects remain elusive [17].

A process implicated in the pathogenesis of CNS disorders is inflammation, a key host defence response to infection and injury. Inflammation is known to contribute to neuronal injury [18], but is also implicated in repair mechanisms in the brain [19]. Achieving the right balance between the damaging and reparative role of inflammation is considered a major target for therapeutic intervention [20]. Although some anti-inflammatory treatments are in clinical trial for acute brain injury, current effective treatments are limited [21, 22]. Finding new therapies with longer time windows and wider therapeutic effects has become a priority. Inflammation in the brain is critically regulated by inflammatory cytokines such as interleukin (IL)-1 and tumour necrosis factor alpha (TNF- $\alpha$ ) that are expressed by, and act on, microglia, astrocytes and endothelial cells [23]. Studies using MSCs delivered to the brain have been carried out $[24,25]$, but no studies have yet assessed the effect of the inflammatory environment on the secretory profile of these locally delivered MSCs.

The MSC secretome can be modulated to boost the beneficial actions of these cells, so that they can respond even more effectively to inflammatory conditions. One way to increase this potential is through priming or preconditioning. Inflammatory priming occurs when a mild (or sub-lethal) inflammatory event induces cellular changes that drive cells towards a more antiinflammatory phenotype, which can eventually lead to a more effective response against future lethal or severe inflammatory events. Different preconditioning treatments have been tested in MSCs in order to induce selected phenotypes [26, 27], but the effect of specific cytokines known to regulate inflammation in the brain or the secretory profile of MSCs has not been widely studied. Here, we tested the hypothesis that priming with inflammatory stimuli would modify the secretome of MSCs towards an anti-inflammatory and trophic phenotype [28]. We show for the first time that MSCs express high constitutive levels of key anti-inflammatory and trophic factors, and that priming with IL-1 triggers secretion of the trophic factor granulocyte-colony stimulating factor (G-CSF), an effect that was only observed in response to IL-1. The addition of IL-1-primed MSC conditioned media (CM) to inflamed microglial cells caused a reduction in the secretion of inflammation markers (IL-6, G-CSF and TNF- $\alpha$ ), and an increase in the microglial-derived anti-inflammatory mediator cytokine IL-10. These results highlight the ability of MSCs to orchestrate other cells to induce a more effective antiinflammatory response, demonstrating the potential use of priming inflammatory treatments to enhance the beneficial actions of MSCs for future stroke therapies.

\section{Methods \\ Human MSCs}

Human bone marrow-derived MSCs were purchased from Lonza (UK) and $3 \mathrm{H}$ Biomedical (Sweden). Three different donors were used in this study: donor 1 (Lonza, 38 years old, male), donor 2 (Lonza, 21 years old, female) and donor 3 (3H Biomedical, 22 weeks old, fetal). Culture flasks (Corning, UK) were coated with $0.1 \%$ gelatin in PBS, overnight at $37{ }^{\circ} \mathrm{C}$, and washed with PBS. MSCs were subsequently cultured in MesenPRO RS medium (Invitrogen, UK) supplemented with 1\% penicillin/streptomycin and $2 \mathrm{mM}$ glutamine. The medium was changed every 4-5 days until cells reached 70-80\% confluency. Cells were then detached with 0.5\% trypsinEDTA (Sigma-Aldrich, UK), counted and split into different tissue culture flasks and further cultured as already described. Cells used for experiments were obtained from culture passages 4-6 and were seeded in gelatin-coated plates at 13,000 cells $/ \mathrm{cm}^{2}, 24 \mathrm{~h}$ prior to treatment.

\section{Differentiation of MSCs and flow cytometry}

Cells obtained from different donors were tested for their ability to differentiate into osteocytes or adipocytes using commercial kits (Millipore, UK), according to the manufacturer's instructions. MSCs were further characterised phenotypically by multicolour flow cytometry 
using the BD Stemflow ${ }^{\mathrm{ma}}$ hMSC Analysis Kit (BD Biosciences, UK) on a FACSVerse flow cytometer (BD Biosciences, UK). The surface markers used for the phenotypic characterisation of MSCs were CD73, CD90, CD105, CD34, CD11b, CD19, CD45 and HLA-DR, as stated by the International Society for Cellular Therapy [29].

\section{Microglial cells (BV2 cells)}

BV2 cells (ATCC, UK), an immortalised murine microglial cell line, were cultured in RPMI-1640 medium (Sigma-Aldrich, UK) supplemented with 10\% FBS (Gibco, UK) and $1 \%$ penicillin/streptomycin (SigmaAldrich, UK) until 70-80\% confluent. Cells were detached with trypsin-EDTA (Sigma-Aldrich, UK), counted and seeded at a density of 13,000 cells $/ \mathrm{cm}^{2}$.

\section{Cell treatments}

\section{MSC priming}

MSC cultures were treated with recombinant human IL- $1 \alpha$, IL- $1 \beta$, TNF- $\alpha$ or interferon gamma (IFN- $\gamma$ ) (all from R\&D Systems UK) at a final, concentration of 1 , 10,50 or $100 \mathrm{ng} / \mathrm{ml}$. After $24 \mathrm{~h}$ of treatment, culture supernatants were collected and analysed for the presence of several cytokines (see "Enzyme-linked immunosorbent assay").

\section{Blocking IL-1 receptor antagonist experiments}

IL-1 receptor antagonist (IL-1Ra) $(200 \mu \mathrm{g} / \mathrm{ml}$, Kineret; Biovitrum, Sweden) was added to MSCs. After $10 \mathrm{~min}$, priming treatments were added normally, without washing (final concentration of $100 \mu \mathrm{g} / \mathrm{ml}$ for IL-1Ra and $10 \mathrm{ng} / \mathrm{ml}$ for inflammatory cytokines). After $24 \mathrm{~h}$, supernatants were collected and analysed.

\section{Conditioned medium treatment of BV2 cells}

MSCs were seeded at a density of $13,000 \mathrm{cells} / \mathrm{cm}^{2}$ in 24-well plates (Corning, UK). Once attached, cells were primed with $10 \mathrm{ng} / \mathrm{ml}$ of human recombinant IL- $1 \alpha$ (R\&D Systems, UK) for $5 \mathrm{~min}$. MSCs were then washed twice with PBS, and fresh MesenPRO medium was added. After $24 \mathrm{~h}$ of incubation, CM were collected. BV2 cells were simultaneously treated with $\mathrm{CM}$ and $1 \mu \mathrm{g} / \mathrm{ml}$ lipopolysaccharide (LPS) from E. coli 0127:B8 (Sigma-Aldrich, UK). Supernatants were collected at $24 \mathrm{~h}$-see detailed experimental protocol (Fig. 1).

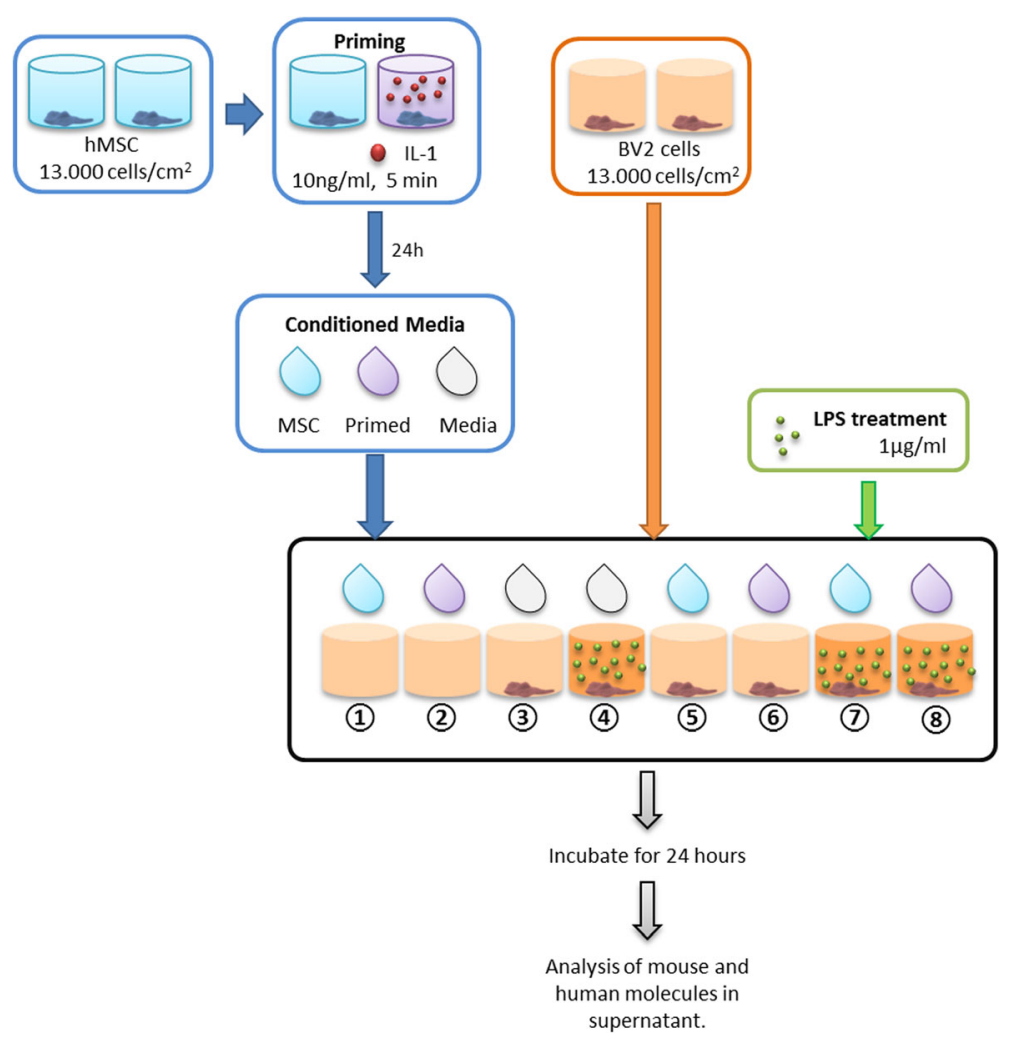

Fig. 1 Experimental protocol of MSC-CM treatment on BV2 cells. MSCs were primed with IL-1a, and the CM used on BV2 cells (treated or not with LPS). Summary of experimental conditions: 1, MSC-CM; 2, primed MSC-CM; 3, untreated BV2; 4, BV2 treated with LPS; 5, BV2 treated with MSC-CM; 6, BV2 treated with primed MSC-CM; 7, BV2 treated with LPS and MSC-CM; 8, BV2 treated with LPS and primed MSC-CM. hMSC human mesenchymal stem/stromal cell, IL interleukin, LPS lipopolysaccharide 


\section{Blocking human G-CSF experiments}

CM from MSCs (primed and unprimed) were incubated in plates previously coated with a neutralising antibody against human G-CSF (R\&D systems, UK). After 2 h of incubation at RT and sterile conditions, CM were collected and added to BV2 cells as already described, and cytokines were measured.

\section{Lactate dehydrogenase assay}

Cell death and proliferation of BV2 cells after cytokine treatments were analysed using a lactate dehydrogenase (LDH) assay kit (Promega, UK) according to the manufacturer's instructions. In brief, to assess cell death, supernatants were collected, LDH was measured and optical densities were normalised to $100 \%$ cell death control. To assess proliferation, all cells were lysed and measured LDH concentrations were compared with control values (untreated BV2 cells). An increase in LDH measurements was interpreted as an increase in cell death or proliferation (respectively).

\section{Enzyme-linked immunosorbent assay}

Levels of human IL-10, brain-derived neurotrophic factor (BDNF), nerve growth factor (NGF), vascular endothelial growth factor (VEGF), TNF- $\alpha$ and G-CSF in culture media from MSCs were quantified by ELISA using DuoSet kits (R\&D Systems, UK) according to the manufacturer's instructions. Human IL-1Ra levels were measured using an ELISA kit from Peprotech (UK) combined with external standards prepared using recombinant human IL-1Ra (National Institute for Biological Standards and Controls (NIBSC), UK). Quantification limits in human ELISAs were $10 \mathrm{pg} / \mathrm{ml}$ for IL-1Ra, $15 \mathrm{pg} / \mathrm{ml}$ for G-CSF, NGF, TNF- $\alpha$ and VEFG, and $25 \mathrm{pg} / \mathrm{ml}$ for BDNF and IL-10. ELISA kits for mouse IL- 6 , TNF- $\alpha$, IL-10 and G-CSF (all quantification limits $\sim 30 \mathrm{pg} / \mathrm{ml}$ ) were purchased from $R \& D$ Systems and used following the manufacturer's instructions. For each assay, samples were diluted as needed and protein levels were calculated against a four-parameter logistic (4-PL) curve fit. All values are expressed as mean \pm standard error of the mean (SEM).

\section{Statistical analysis}

In each experiment, a minimum of four independent cultures were included. Graphs, 4-PL curves and statistical analysis were done using GraphPad Prism software version 7 for Windows (CA, USA). Treatment effects in each donor were assessed by nonparametric one-way ANOVA analysis. BV2 data were analysed by parametric one-way ANOVA. Fisher post- hoc tests were only performed if statistical significance was achieved $(p<0.05)$.

\section{Results}

Phenotypic characterisation of human MSCs in vitro

MSCs derived from three donors were differentiated successfully into adipocytes and osteocytes, evidenced by the presence of lipid droplets (stained in red, Fig. 2a) and calcium deposits (Fig. 2b), respectively. Different antibodies and corresponding isotype controls were used to assess expression of specific MSCpositive cell surface markers [29]. MSCs derived from different donors were, on average, 98.65\% CD90positive (Fig. 2c), 98.17\% CD73-positive (Fig. 2d) and 91.50\% CD105-positive (Fig. 2e). Less than $2 \%$ of the cells were positive for CD34, CD11b, CD45, CD19 and HLA-DR (Fig. 2f, Table 1). FACS analysis also indicated the presence of single-cell populations, with a low amount of debris, duplets and triplets, indicating a high proportion of normal and healthy cells (data not shown).

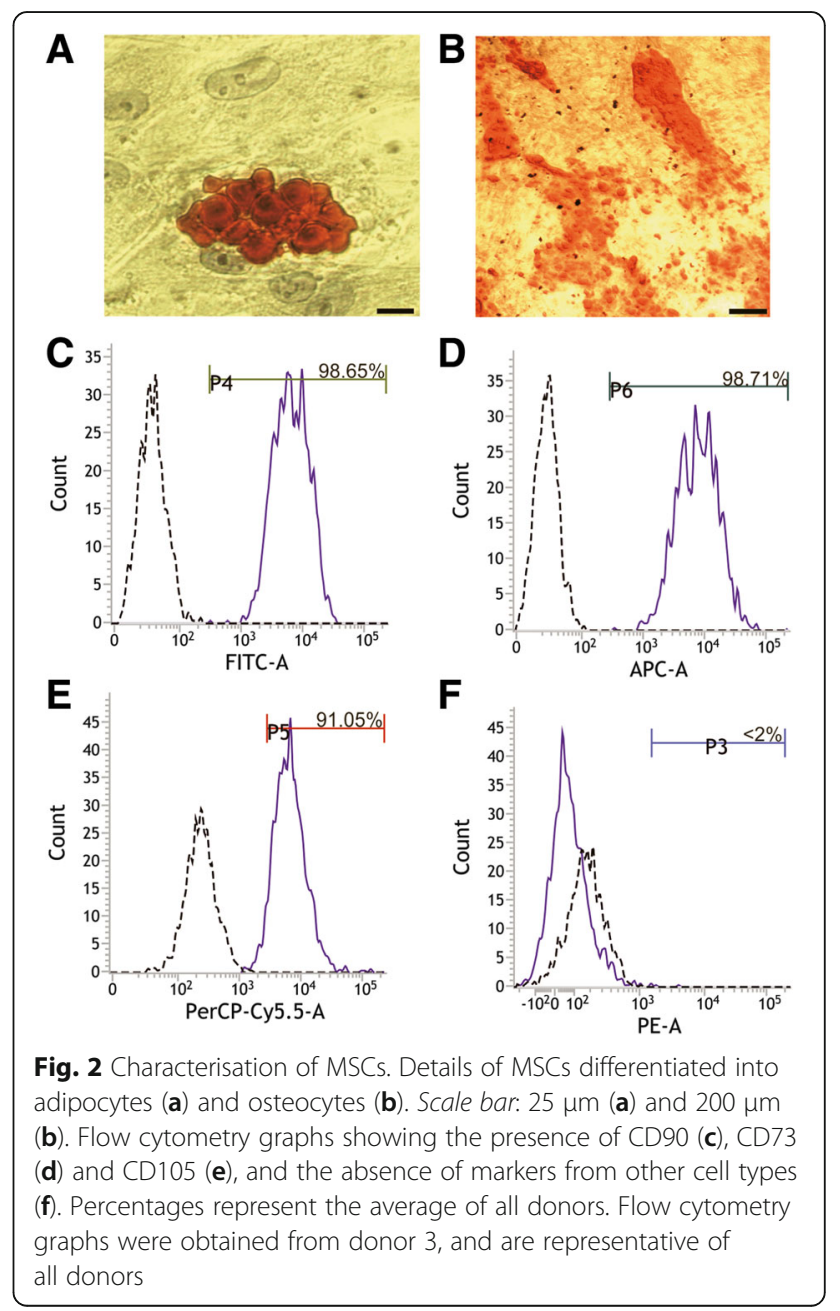


Table 1 Phenotyping characterisation of MSCs for all markers and all donors

\begin{tabular}{lcllr}
\hline & Donor 1 & Donor 2 & Donor 3 & Mean \pm SEM \\
\hline CD90 & 95.97 & 100 & 100 & $98.65 \pm 1.10$ \\
CD73 & 94.76 & 99.91 & 99.86 & $98.17 \pm 1.39$ \\
CD105 & 80.24 & 99.48 & 94.79 & $91.50 \pm 4.73$ \\
Other & 3.23 & 2.07 & 0.21 & $1.83 \pm 0.72$ \\
\hline
\end{tabular}

Quantification of marker expression from three independent cultures, expressed as the mean \pm SEM

MSC human mesenchymal stem/stromal cell

\section{MSCs secrete basal levels of anti-inflammatory and} neurotrophic mediators

MSCs obtained from different donors were expanded and cultured, and their media were analysed for the presence of anti-inflammatory cytokines and trophic factors under basal conditions by ELISA (all values presented are expressed as mean \pm SEM). MSCs constitutively expressed BDNF, IL-1Ra, NGF, VEGF, GCSF and IL-10 (Fig. 3), although the levels secreted varied between donors; MSCs from donors 1 and 3 secreted moderate concentrations of BDNF $(66.5 \pm 3.6 \mathrm{pg} / \mathrm{ml}$ and $62.6 \pm 4.7 \mathrm{pg} / \mathrm{ml}$, respectively), while donor 2 only secreted $6.2 \pm 0.9 \mathrm{pg} / \mathrm{ml} \mathrm{BDNF}$ (Fig. 3a). In contrast, cells from donor 2 secreted the highest concentration of NGF $(11.0 \pm 7.1 \mathrm{pg} / \mathrm{ml})(1.2 \pm 1.0 \mathrm{pg} / \mathrm{ml}$ in donor 1 and $3.4 \pm$ $4.0 \mathrm{pg} / \mathrm{ml}$ in donor 3; Fig. 3b).

Concentrations of G-CSF were also variable between donors, with low levels secreted in all donors (not detectable in donor $1,38.3 \pm 7.9 \mathrm{pg} / \mathrm{ml}$ in donor 2 and 6.7 $\pm 4.7 \mathrm{pg} / \mathrm{ml}$ in donor 3; Fig. 3c). The levels of IL-10
(Fig. 3d) were similar in all three donors $(13.9 \pm 11.1 \mathrm{pg} /$ $\mathrm{ml}, 14.1 \pm 11.5 \mathrm{pg} / \mathrm{ml}$ and $15.7 \pm 9.7 \mathrm{pg} / \mathrm{ml}$, respectively). Other factors such as VEGF were secreted in high amounts in cells from donor $1(1182.3 \pm 128.5 \mathrm{pg} / \mathrm{ml})$; levels were lower in the other donors (donor 2, $159.3 \pm$ $17.7 \mathrm{pg} / \mathrm{ml}$ and donor 3, $247.0 \pm 55.6 \mathrm{pg} / \mathrm{ml}$; Fig. 3e). The protein with the highest secretion in all three donors was IL-1Ra, which was in the nanogram range $(0.79 \pm 0.1 \mathrm{ng} / \mathrm{ml}$ in donor $1,2.4 \pm 0.4 \mathrm{ng} / \mathrm{ml}$ in donor 2$)$, being especially high in the youngest donor (donor 3, $22.4 \pm 4.9 \mathrm{ng} / \mathrm{ml}$; Fig. 3f).

\section{IL-1 selectively primes MSCs to produce high levels of anti-inflammatory and pro-trophic factors}

Basal concentrations of mediators were assessed in the supernatant of MSCs treated with increasing concentrations of IL- $1 \alpha$, IL-1 $\beta$, TNF- $\alpha$ or IFN- $\gamma$ for $24 \mathrm{~h}$. Whilst TNF- $\alpha$ or IFN- $\gamma$ had no effect on secretion of G-CSF from MSCs derived from the three donors (Fig. 4a, b), IL- $1 \alpha$ and IL-1 $\beta$ induced strong G-CSF release from MSCs obtained from all of the donors (Fig. 4c, d). The magnitude of this response was different in each donor, with the highest increase observed in MSCs obtained from donor 3 (ranging from $5.9 \pm 3.6 \mathrm{pg} / \mathrm{ml}$ in basal conditions to $6.8 \pm 1.7 \mathrm{ng} / \mathrm{ml}$ after IL- $1 \alpha$ and $7.4 \pm 2.1 \mathrm{ng} / \mathrm{ml}$ after IL-1 $\beta)$. In contrast, IL- $1 \alpha$ and IL-1 $\beta$ had no effect on VEGF, NGF or IL-1Ra expression (Additional file 1: Figure S1). Increased IL-10 levels were observed after IL- $1 \alpha$ and IL- $1 \beta$ treatments, although this was not significant due to high variability. BDNF levels in response to IL- $1 \alpha$ and IL-1 $\beta$ were highly variable with cells from
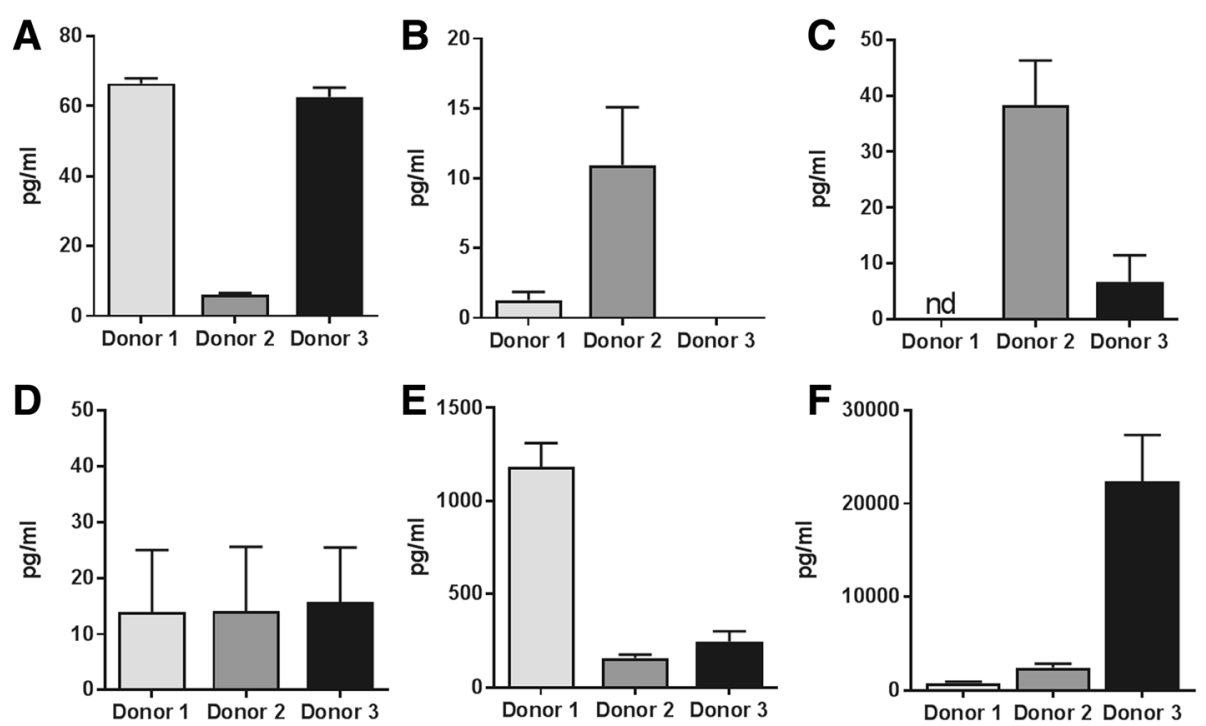

Fig. 3 Constitutive secretion. MSCs express several anti-inflammatory cytokines and trophic factors under basal conditions ( $n=3$ experiments/ donor), as measured by ELISA. Measurements of BDNF (a), NGF (b), G-CSF (c), IL-10 (d), VEGF (e) and IL-1Ra (f) in MSCs derived from the three different donors. BDNF brain-derived neurotrophic factor, G-CSF granulocyte-colony stimulating factor, IL interleukin, IL-1Ra interleukin-1 receptor antagonist, nd not detectable, NGF nerve growth factor, VEGF vascular endothelial growth factor 

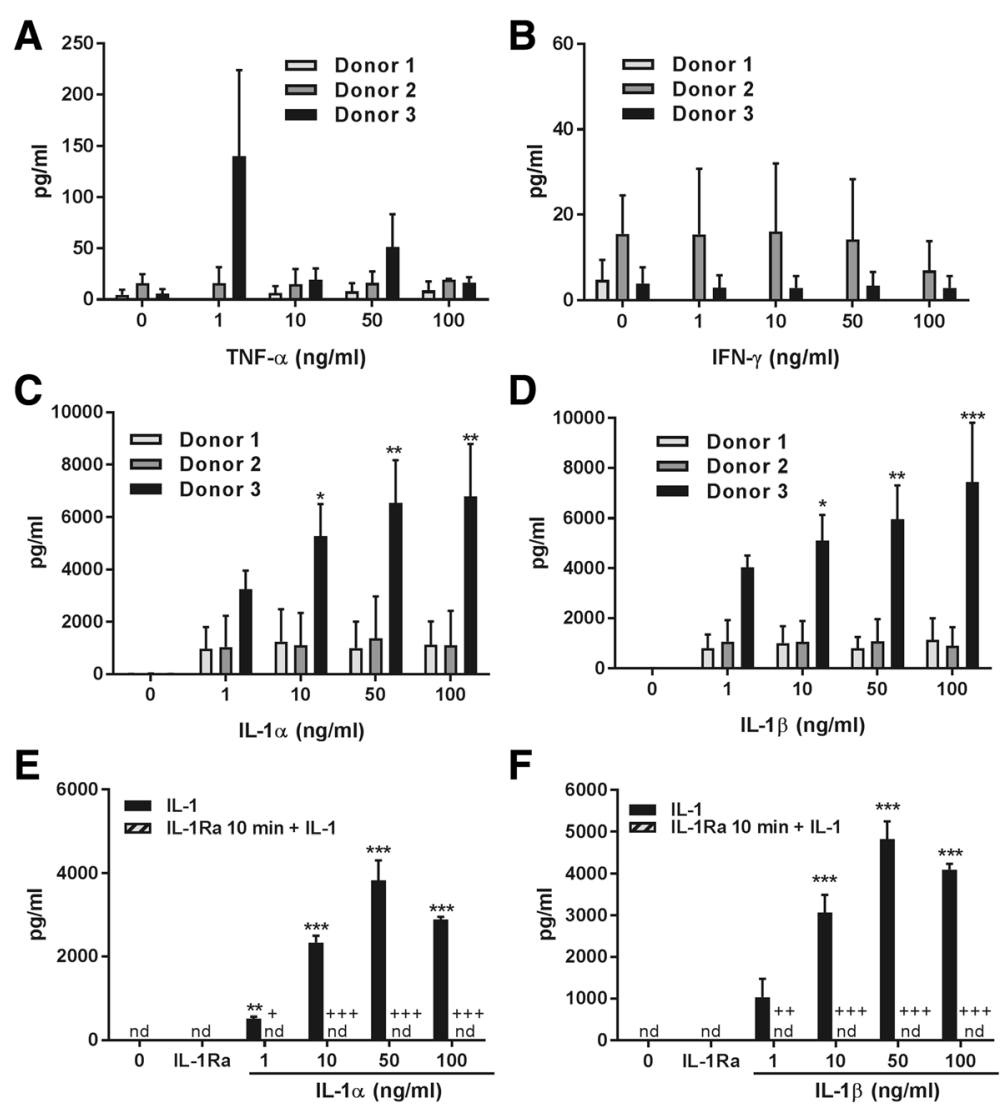

Fig. 4 Secretion of G-CSF by MSCs. Levels of G-CSF were assayed after treating cells from different donors ( $n=3$ experiments/donor) with different concentrations of TNF-a (a) and IFN- $\boldsymbol{(}$ (b) for $24 \mathrm{~h}$, which had no significant effect on the secretion of G-CSF. Treatments with IL-1a (c) and IL-1 $13(\mathbf{d})$ for $24 \mathrm{~h}$ induced a strong increase in the secretion of G-CSF. The youngest donor presented the highest increase in secretion, as measured by ELISA ( ${ }^{*} p<$ $0.05,{ }^{* *} p<0.01,{ }^{* * *} p<0.001$ vs untreated from same donor), despite all donors showing an increase in the secreted levels of G-CSF. The increased secretion of G-CSF in response to priming with IL-1a (e) and IL-1 1 (f) is completely inhibited when IL 1-Ra $(100 \mu \mathrm{g} / \mathrm{ml})$ is added $10 \mathrm{~min}$ prior to the treatment. ${ }^{*} p<0.05,{ }^{* * *} p<0.001$ vs untreated; ${ }^{++} p<0.05,{ }^{+++} p<0.001 \mathrm{IL}-1$ Ra condition vs cytokine treatment only. Cells from donor 3 were used in the inhibition experiment. G-CSF granulocyte-colony stimulating factor, IFN- $y$ interferon gamma, IL interleukin, IL-1Ra interleukin-1 receptor antagonist, nd not detectable, TNF-a tumour necrosis factor alpha

the three donors showing increased, decreased or unaltered BDNF levels after IL- $1 \alpha$ and IL- $1 \beta$ treatments (Additional file 1: Figure S1). Finally, release of VEGF, NGF, IL-1Ra, IL-10 and BDNF was unaltered by treatments with TNF- $\alpha$ or IFN- $\gamma$ (Additional file 2: Figure S2, only a significant decrease in VEGF was detected in donor 1). Because cells from donor 3 produced the highest basal level of IL-1Ra and showed the most robust increase in the secretion of G-CSF in response to IL-1, all further experiments were carried out using cells from this donor.

IL-1 acts normally through IL-1 receptor type 1 (IL-1R1) [30]. To assess whether the actions of IL-1 on MSCs occurred via actions on IL-1R1, we tested the effect of human recombinant IL-1Ra (added prior to treatment with IL-1) on G-CSF release from IL-1primed MSCs. IL-1Ra added before treatment with IL- $1 \alpha$ or IL- $1 \beta$ significantly and completely blocked the priming effect of IL-1 on G-CSF secretion $(p<$
0.01-0.001; Fig. 4e, f), thus confirming that IL-1 actions were mediated by IL-1R1 activity.

Anti-inflammatory effect of conditioned medium of MSCs on LPS-treated microglial cells is potentiated by priming of MSCs with IL-1

To test the hypothesis that MSCs can exert antiinflammatory properties and could therefore be used as potent anti-inflammatory agents, we added CM from untreated or IL-1-primed MSCs to LPS-treated BV2 cells. After priming with IL-1 $\alpha$ (see scheme in Fig. 1), MSCs exhibited increased secretion of IL-6 $(p<0.01$; Fig. 5a) and G-CSF (Fig. 5c), whilst TNF- $\alpha$ and IL-10 remained undetectable (Fig. 5b, d). Stimulation of BV2 cells with LPS induced microglial activation, measured by increased secretion of IL-6 ( 170 to $\sim 2000 \mathrm{pg} / \mathrm{ml}, p<$ 0.001; Fig. 5e), TNF- $\alpha(\sim 1800 \mathrm{pg} / \mathrm{ml}, p<0.001$; Fig. $5 \mathrm{f})$ and G-CSF $(\sim 10,000$ pg/ml, $p<0.001$; Fig. 5g). 

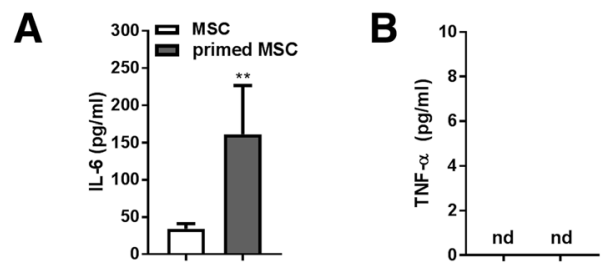

$\mathbf{E}$

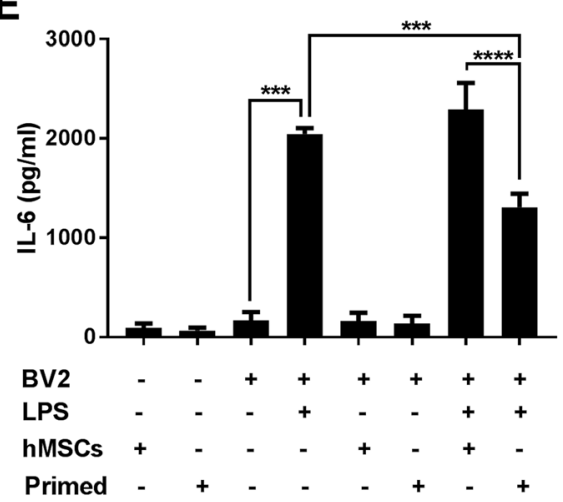

G

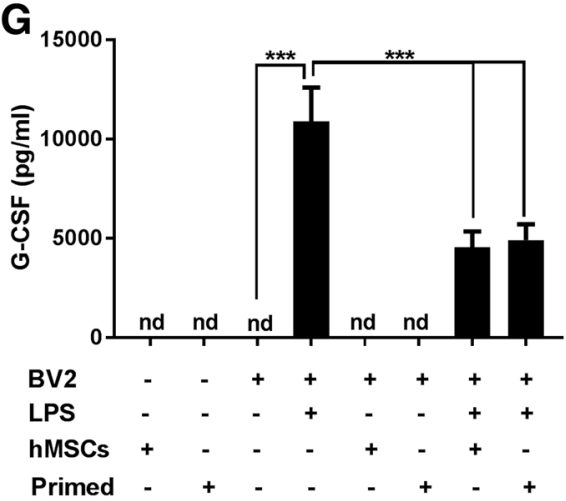

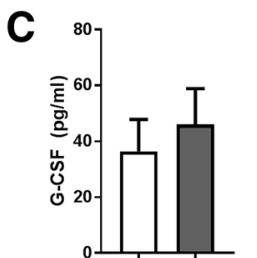

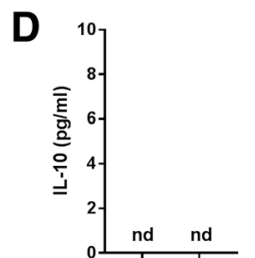

F

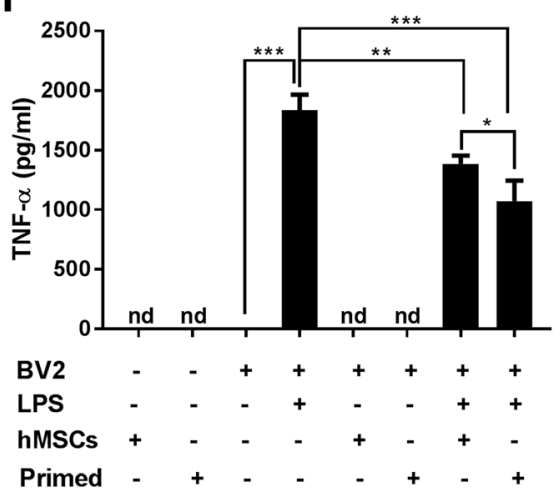

H

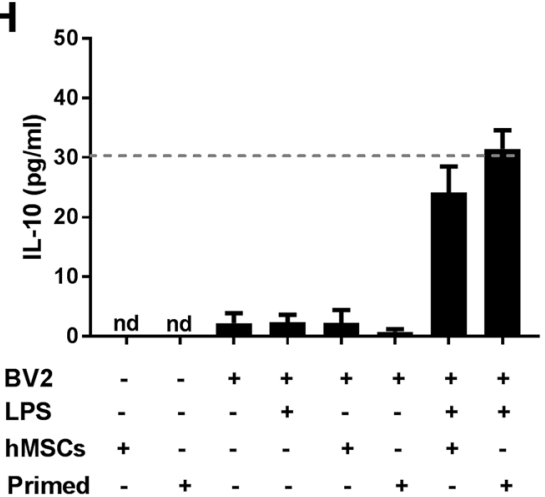

Fig. 5 BV2 cell treatment with MSC-CM. Concentrations of human IL-6 (a, ${ }^{* *} p<0.01$ primed vs unprimed), TNF-a (b), G-CSF (c) and IL-10 (d) were measured in the CM of MSCs ( $n=4$ experiments). ELISAs to detect mouse isoforms of the same molecules (e, $\mathbf{f}, \mathbf{g}$, and $\mathbf{h}$, respectively, $n=4$ experiments) showed that the addition of MSC-CM induced significant changes in their secretion (when compared with LPS-treated BV2 cells). CM from IL-1a-primed MSCs induced a stronger response, evidenced by a larger reduction in the secretion of IL-6 $(p<0.001)$ and TNF-a $(p<0.05)$ and by an increased secretion of IL-10. Note that most IL-10 values are below the quantification limit of the assay (dashed line), so statistical analysis cannot be performed with these values. ${ }^{*} p<0.05,{ }^{* *} p<0.01,{ }^{* * *} p<0.001$, ${ }^{* * *} p<0.001$. G-CSF granulocyte-colony stimulating factor, hMSC human mesenchymal stem/stromal cell, IFN- $y$ interferon gamma, IL interleukin, IL-1Ra interleukin-1 receptor antagonist, LPS lipopolysaccharide, nd not detectable, TNF-a tumour necrosis factor alpha

The addition of MSC-CM to LPS-treated BV2 microglial cells did not alter the secretion of IL-6 (Fig. 5e), but induced a significant reduction in TNF- $\alpha(\sim-25 \%, p<$ $0.01)$ and G-CSF ( $55 \%$ reduction, $p<0.001)$, as well as a marked increase in secreted IL-10 (12-fold increase, from $\sim 2$ to $\sim 24 \mathrm{pg} / \mathrm{ml}$ ). These changes were more pronounced when LPS-treated BV2 cells were exposed to CM from IL- $1 \alpha$-primed MSCs, as shown by a significant reduction in IL-6 secretion $(\sim-35 \%, p<0.001$ vs LPS, $p<$ 0.0001 vs untreated CM; Fig. 5e) and in TNF- $\alpha(-41 \%$, $p<0.001$ vs LPS, $p<0.05$ vs untreated CM; Fig. 5f) and a greater increase in IL-10 secretion (13-fold increase, $\sim 30 \mathrm{pg} / \mathrm{ml}$, vs untreated CM; Fig. 5h). The reduction in the secretion of G-CSF was unaffected by priming of
MSCs with IL- $1 \alpha(\sim 55 \%$ reduction, $p<0.001$ vs LPS treated BV2; Fig. 5g). CM from MSCs primed with TNF- $\alpha$ and IFN- $\gamma$ were not effective at reducing the secretion of inflammatory markers (data not shown). CM (from untreated or primed MSCs) added to untreated BV2 cells had no effect on any of the mediators tested, thus discarding an unspecific effect of the addition of media from a different cell type. We measured both human and mouse isoforms of these mediators in supernatants from all conditions, and no cross-reactivity between species was observed. Finally, none of the treatments induced significant cell death or proliferation in BV2 microglial cultures (Additional file 3: Figure S3). 
Given the large amount of G-CSF secreted by MSCs in response to IL-1 preconditioning, and the fact the anti-inflammatory effect of MSC-CM was significantly enhanced by IL-1 priming, we next hypothesised that GCSF could be a key mediator involved in these antiinflammatory actions. To this end, we found that the levels of mouse IL- 6 and TNF- $\alpha$ were significantly higher when human G-CSF was neutralised with a specific antibody (Fig. 6), thus confirming its role in the modulation of the secretion of these two molecules for anti-inflammatory actions on BV2 cells.

\section{Discussion}

We describe the priming of MSCs with inflammatory cytokines to test whether this causes a secretory profile towards an anti-inflammatory and pro-trophic phenotype. We show that treatment of MSCs with IL- $1 \alpha$ or IL-1 $\beta$ increased the secretion of trophic factors such as G-CSF. This was a specific effect of IL-1, because TNF- $\alpha$ and IFN- $\gamma$ failed to induce this response.

In order to test the cells' anti-inflammatory and protrophic potential, we added CM from MSCs to inflamed murine BV2 cells. Priming of MSCs induced the secretion of anti-inflammatory mediators such as IL-10 in BV2 cells, as well as a decrease in the secretion of proinflammatory cytokines (IL-6, TNF- $\alpha$ ). These effects were more marked when MSCs had been primed previously with IL-1, supporting the potential use of priming treatments to induce more desirable phenotypes. We also confirmed that IL-1 exerts these effects via IL-1R1, because IL-1Ra completely blocked the increase in G-CSF after the priming. Furthermore, inhibition experiments using a specific human G-CSF neutralising antibody demonstrated that G-CSF is critical for the anti-inflammatory effect of MSCs triggered by IL-1 priming.

While first identified as a growth factor that promotes survival, proliferation and differentiation of myeloid progenitors [31], G-CSF has since been found to be a key neurotrophic factor in the CNS [32]. The G-CSF receptor is expressed throughout the adult CNS and its activation includes inhibition of apoptosis in neurons and initiation of neurogenesis in neural stem cells [33, 34].

Potentially beneficial actions of G-CSF reported in animal models include increased synaptogenesis, angiogenesis, neuroprotection, neurogenesis, plasticity and anti-apoptosis [34, 35]. In rodent models of cerebral ischaemia, G-CSF treatment has been shown to have neuroprotective effects leading to reduced infarct volume $[36,37]$, improved functional recovery $[34,38]$ and neurogenesis [39]. G-CSF has also been described as a mobilisation factor of endogenous or transplanted MSCs [40, 41], which along with all of the aforementioned features could explain some sensorimotor and functional improvements already described with MSC treatment [42, 43]. Moreover, the increased secretion of G-CSF induced by MSCs induced an M2 or M2-like functional phenotype in macrophages (which implies an anti-inflammatory and proregenerative phenotype) [44-46], as well as leading to higher rates of tissue remodelling and angiogenesis [47].

There is some controversy in the literature over the effects of G-CSF on experimental ischaemic stroke and brain haemorrhage [48], with positive or neutral outcomes as well as some negative results being reported $[49,50]$. Clinical trials showed that the administration of G-CSF to stroke patients is safe (ClinicalTrials.gov NCT00901381, NCT00132470), but the data on efficacy have been contradictory [51] (ClinicalTrials.gov NCT009278361, NCT00132470). A study has suggested that G-CSF could be more beneficial when administered in the chronic phase to potentiate neural repair mechanisms [48]. This potential efficacy in delayed treatments favours the hypothesis that administration of cell therapies in the chronic phase leads to remodelling and repair of the injured brain. Other clinical trials assessing the safety and efficacy of G-CSF in Alzheimer's disease (ClinicalTrials.gov NCT01617577), ALS (ClinicalTrials.gov NCT01825551),
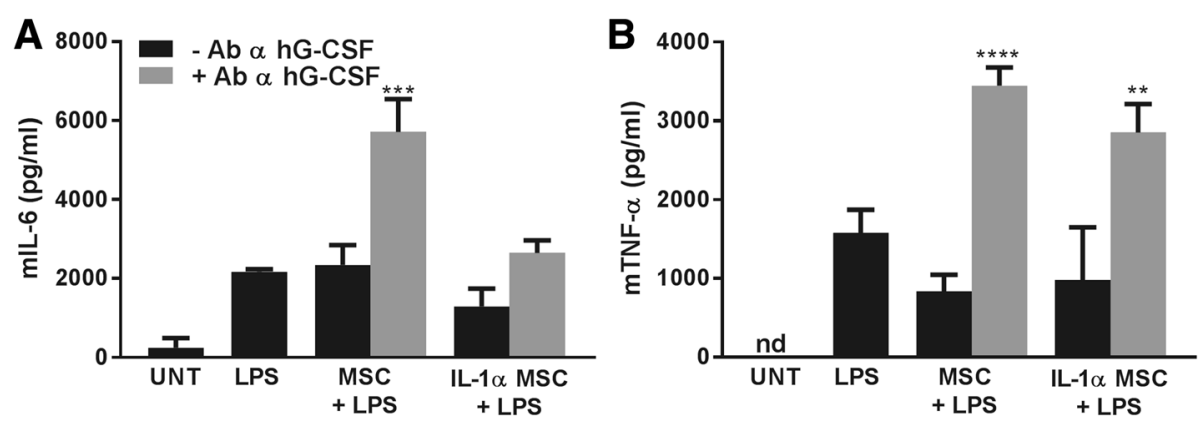

Fig. 6 G-CSF neutralisation of MSC-CM induces raised IL-6 and TNF-a levels in BV2 cells. Addition of MSC-CM depleted of G-CSF induced higher secretion of IL-6 (a) and TNF-a (b) from LPS-stimulated BV2 cells ( $p<0.001$ for IL-6; $p<0.0001$ and $p<0.01$ vs non-neutralised CM, for TNF-a when CM was from unprimed or primed MSCs, respectively). G-CSF granulocyte-colony stimulating factor, $h$ human, MSC mesenchymal stem/stromal cell, IL interleukin, LPS lipopolysaccharide, nd not detectable, TNF-a tumour necrosis factor alpha 
brain injury and other neurodegenerative diseases (ClinicalTrials.gov NCT02236065) are being conducted.

G-CSF may play a dual roleas it can also be produced as an autocrine protective mechanism, because neurons secrete it in ischemic conditions in an attempt to reduce neuronal apoptosis $[34,52]$. In this study we have shown that MSC priming with IL-1 induces increased secretion of G-CSF (in MSCs), whilst G-CSF secretion is reduced in LPS-treated BV2 cells exposed to MSC-CM, demonstrating the dual role of G-CSF. In this case, the reduction in the secretion of G-CSF by BV2 cells might indicate that BV2 cells were responding more effectively to the inflammation.

The increase in IL-10 in BV2 cells after MSC-CM treatment adds potential to the therapeutic application of MSCs, because IL-10 is known to inactivate macrophages and to trigger matrix deposition and tissue remodelling $[45,46]$. MSCs can be primed to secrete trophic factors, and they can also induce changes in the secretory phenotype of other cells, and could potentially increase the chances for injured or inflamed tissue to achieve a better recovery after an inflammatory event. In agreement with this, our data demonstrated that MSCs regulate microglial cells to secrete less inflammatory mediators and more anti-inflammatory cytokines, which may contribute to the healing and repair of the tissue.

We also need to consider the constitutive secretion of a wide variety of other cytokines. MSCs constitutively secrete high concentrations of IL-1Ra, and this is of interest because it has been shown in humans that IL$1 \mathrm{Ra}$ is required to be administrated repeatedly and in high doses to maintain its efficacy [53]. Therefore, MSCs from certain donors (those with a high constitutive secretion) may become a cellular system to deliver high and sustained doses of IL-1Ra. Besides, because MSCs tend to migrate to injured, inflamed or ischaemic areas [5-7], this would be a targeted delivery. This would imply a significant improvement from actual therapies, which require repeated doses of expensive recombinant human proteins [53, 54]. Other molecules that are expressed constitutively could possibly be stimulated by other priming agents not tested here.

Our results highlighted variability between MSCs from different donors, but a clear correlation between their secretome and sources (sex and age) was not found. Similarly to other studies $[55,56]$, we could not observe any clear relationship between the levels of these cytokines and the age or the sex of the donor. Variability between donors has been reported widely in the literature, and can be observed at the gene expression level $[57,58]$ and in response to differentiation stimuli $[55,58,59]$. When describing the secretome of MSCs, results are highly variable between studies, indicating once more the important variability of these cells and the existence of subpopulations [60-62]. This variability needs to be considered when designing new cell therapies because it can limit applicability.

\section{Conclusions}

Stem cell therapies using MSCs are a promising option for the treatment of several neurological conditions because of their safety, their immunosuppressive properties and their ability to sense and reach the inflamed area, therefore potentially improving recovery and repair $[1,2,6,10]$. In our study, we demonstrate that preconditioning treatments increased MSC secretion of anti-inflammatory mediators and trophic factors [8]. They can also trigger changes in other cells, switching them towards a more anti-inflammatory and pro-regenerative phenotype. These results highlight the possibility of modulating the secretome of MSCs, and confirm the beneficial actions they can exert when added to inflamed cells or tissues. Taken together, these results propose MSCs as an excellent candidate to be considered when designing more effective cell therapies to be used in CNS inflammatory conditions.

\section{Additional files}

Additional file 1: Figure S1. Is showing levels of cytokines secreted by MSCs from three different donors after IL-1a or IL-1 $1 \beta$ treatment. Secretion of VEGF (A, B) and NGF (C, D) was not modified by any treatments in any of the donors, but secretion of IL-10 showed a non-significant increase in some donors (E, F). Levels of IL-1 Ra were high and unchanged after IL-1 treatments $(\mathbf{G}, \mathbf{H})$. Changes in the levels of BDNF were different in each donor, showing significance in some donors $(\mathbf{I}, \mathbf{J})(n=3$ experiments/ donor). ${ }^{*} p<0.05,{ }^{* *} p<0.01$, ${ }^{* * *} p<0.001$ vs untreated. (TIF $461 \mathrm{~kb}$ )

Additional file 2: Figure S2. Is showing levels of cytokines secreted by MSCs from three different donors, after TNF-a or IFN- $\gamma$ treatment. Secretion of VEGF (A, B), NGF (C, D), IL-10 (E, F), IL-1Ra (G, H) and BDNF (I, J). Donor 1 showed a significant decrease in the amount of VEGF, but donors 2 and 3 showed no response. No significant changes were detected in secretion of NGF, IL-10, IL-1Ra and BDNF ( $n=3$ experiments/donor). ${ }^{*} p<0.05,{ }^{* *} p<0.01$, *** $p<0.001$ vs untreated. (TIF $470 \mathrm{~kb}$ )

Additional file 3: Figure S3. Is showing measurement of cell death and proliferation of BV2 cells in CM treatment experiments. $\mathrm{LDH}$ was measured in supernatants (A) and cell lysates (B) as indirect measurements of cell death and proliferation. None of the treatments induced significant cell death or proliferation. (TIF $585 \mathrm{~kb}$ )

\section{Abbreviations}

BDNF: Brain-derived neurotrophic factor; CM: Conditioned media; CNS: Central nervous system; ELISA: Enzyme-linked immunosorbent assay; IFN-ү: Interferon gamma; IL: Interleukin; IL-1R1: Interleukin-1 receptor type 1; IL-1Ra: Interleukin-1 receptor antagonist; LDH: Lactate dehydrogenase; MSC: Mesenchymal stem/stromal cell; nd: Not detectable; NGF: Nerve growth factor; SEM: Standard error mean; TNF-a: Tumour necrosis factor alpha; VEGF: Vascular endothelial growth factor

\section{Acknowledgements}

The authors would like to acknowledge the assistance of Rebecca Holley (Wellcome Trust Centre for Cell-Matrix Research, UK Centre for Tissue Engineering) and Gareth Howell (Flow Cytometry Facility within the Manchester Collaborative Centre for Inflammation Research). They thank Swedish Orphan Biovitrum AB for providing the Kineret ${ }^{\oplus}$ used in these studies. 


\section{Funding}

The work was supported by funds from the Stroke Association (UK), the Engineering and Physical Sciences Research Council (EPSRC), the Medical Research Council (MRC), the Centre for Doctoral Training in Regenerative Medicine studentship grant EP/L014904/1 and the Manchester Regenerative Medicine Network (MaRM).

\section{Availability of data and materials}

The datasets generated during and/or analysed during the current study are available from the corresponding author on reasonable request.

\section{Authors' contributions}

ER-C and CC were responsible for the collection and assembly of data, data analysis and interpretation, and manuscript writing. JM, LM and SA-A were responsible for the collection and/or assembly of data, data analysis and interpretation. NJR, CMK, SMA and EP were responsible for conception and design, acquisition of funding and revision of the manuscript. All authors read and approved the final manuscript.

\section{Competing interests}

The authors declare that they have no competing interests.

\section{Consent for publication}

All authors have approved the submission for publication.

\section{Ethics approval and consent to participate}

Not applicable.

\section{Publisher's Note}

Springer Nature remains neutral with regard to jurisdictional claims in published maps and institutional affiliations.

\section{Author details}

${ }^{1}$ Faculty of Biology, Medicine and Health, University of Manchester, Manchester, UK. 'Wellcome Trust Centre for Cell-Matrix Research, Faculty of Biology, Medicine and Health, University of Manchester, Manchester, UK.

Received: 1 November 2016 Revised: 1 March 2017

Accepted: 8 March 2017 Published online: 17 April 2017

\section{References}

1. Giordano A, Galderisi U. From the laboratory bench to the patient's bedside: an update on clinical trials with mesenchymal stem cells. J Cell Physiol. 2006;211:27-35.

2. Bang OY, Lee JS, Lee PH, et al. Autologous mesenchymal stem cell transplantation in stroke patients. Ann Neurol. 2005:57(6):874-82.

3. Muñoz-Elias G, Woodbury D, Black I. Marrow stromal cells, mitosis, and neuronal differentiation: stem cell and precursor functions. Stem Cells. 2003; 21:437-48.

4. Pittenger MF. Multilineage potential of adult human mesenchymal stem cells. Science. 1999;284(5411):143-7.

5. Reagan MR, Kaplan DL. Concise review: Mesenchymal stem cell tumorhoming: detection methods in disease model systems. Stem Cells. 2011 29(6):920-7.

6. Le Blanc K, Pittenger MF. Mesenchymal stem cells: progress toward promise. Cytotherapy. 2005;7(1):36-45

7. Simard AR, Rivest S. Bone marrow stem cells have the ability to populate the entire central nervous system into fully differentiated parenchymal microglia. FASEB J. 2004;18(9):998-1000.

8. Caplan Al, Correa D. The MSC: an injury drugstore. Cell Stem Cell. 2011;9(1): $11-5$.

9. Chen J, Li Y, Wang $L$, et al. Therapeutic benefit of intravenous administration of bone marrow stromal cells after cerebral ischemia in rats. Stroke. 2001:32(4):1005-11.

10. Kalladka D, Muir KW. Brain repair: cell therapy in stroke. Stem Cells Cloning 2014;7:31-44.

11. Torres-Espín A, Hernández J, Navarro X. Gene expression changes in the injured spinal cord following transplantation of mesenchymal stem cells or olfactory ensheathing cells. PLOS ONE. 2013;8(10):e76141.

12. Joyce N, Annett $G$, Wirthlin L, et al. Mesenchymal stem cells for the treatment of neurodegenerative disease. Regen Med. 2011;5(6):933-46.
13. Lalu MM, McIntyre L, Pugliese C, et al. Safety of cell therapy with mesenchymal stromal cells (SafeCell): a systematic review and meta-analysis of clinical trials. PLOS ONE. 2012;7(10):e47559.

14. Chen M, Li X, Zhang $X$, et al. The inhibitory effect of mesenchymal stem cell on blood-brain barrier disruption following intracerebral hemorrhage in rats: contribution of TSG-6. J Neuroinflamm. 2015;12(1):1-14.

15. Boido M, Piras A, Valsecchi V, et al. Human mesenchymal stromal cell transplantation modulates neuroinflammatory milieu in a mouse model of amyotrophic lateral sclerosis. Cytotherapy. 2014;16(8):1059-72.

16. Kim S, Chang K-A, Kim JA, et al. The preventive and therapeutic effects of intravenous human adipose-derived stem cells in Alzheimer's disease mice. PLoS ONE. 2012;7(9):e45757.

17. Dazzi F, Horwood NJ. Potential of mesenchymal stem cell therapy. Curr Opin Oncol. 2007;19(6):650-5.

18. Tobin MK, Bonds JA, Minshall RD, et al. Neurogenesis and inflammation after ischemic stroke: what is known and where we go from here. J Cereb Blood Flow Metab. 2014;34(10):1573-84.

19. Borsini A, Zunszain PA, Thuret $\mathrm{S}$, et al. The role of inflammatory cytokines as key modulators of neurogenesis. Trends Neurosci. 2014;38(3):145-57.

20. Reardon S. A world of chronic disease. Science. 2011;333(6042):558-9.

21. Donnan GA, Fisher M, Macleod M, et al. Stroke Lancet. 2008;371(9624): 1612-23.

22. George PM, Steinberg GK. Novel stroke therapeutics: unraveling stroke pathophysiology and its impact on clinical treatments. Neuron. 2015;87(2): 297-309.

23. Huang J, Upadhyay UM, Tamargo RJ. Inflammation in stroke and focal cerebral ischemia. Surg Neurol. 2006;66(3):232-45.

24. Xiao J, Nan Z, Motooka Y, et al. Transplantation of a novel cell line population of umbilical cord blood stem cells ameliorates neurological deficits associated with ischemic brain injury. Stem Cells Dev. 2005;14(6):722-33.

25. Kurozumi K, Nakamura K, Tamiya T, et al. BDNF gene-modified mesenchymal stem cells promote functional recovery and reduce infarct size in the rat middle cerebral artery occlusion model. Mol Ther. 2004;9(2): 189-97.

26. Kim SJ, Moon GJ, Chang WH, et al. Intravenous transplantation of mesenchymal stem cells preconditioned with early phase stroke serum: current evidence and study protocol for a randomized trial. Trials. 2013; 14(1):1.

27. Li Q, Wang Y, Deng Z. Pre-conditioned mesenchymal stem cells: a better way for cell-based therapy. Stem Cell Res Ther. 2013:4(3):1.

28. Trento C, Dazzi F. Mesenchymal stem cells and innate tolerance: biology and clinical applications. Swiss Med Wkly. 2010. doi:10.4414/smw.2010. 13121.

29. Dominici M, Le Blanc K, Mueller I, et al. Minimal criteria for defining multipotent mesenchymal stromal cells. The International Society for Cellular Therapy position statement. Cytotherapy. 2006;8(4):315-7.

30. Qian J, Zhu L, Li Q, et al. Interleukin-1R3 mediates interleukin-1-induced potassium current increase through fast activation of Akt kinase. Proc Natl Acad Sci U S A. 2012:109(30):12189-94.

31. Welte K. Discovery of G-CSF and Early Clinical Studies. Twenty Years of GCSF. Basel: Springer Basel; 2012. p. 15-24.

32. Diederich K, Sevimli S, Dörr H, et al. The role of granulocyte-colony stimulating factor (G-CSF) in the healthy brain: a characterization of G-CSFdeficient mice. J Neurosci. 2009;29(37):11572-81.

33. Kirsch F, Krüger C, Schneider A. The receptor for granulocyte-colony stimulating factor (G-CSF) is expressed in radial glia during development of the nervous system. BMC Dev Biol. 2008:8(32):32.

34. Schneider A, Krüger C, Steigleder T, et al. The hematopoietic factor G-CSF is a neuronal ligand that counteracts programmed cell death and drives neurogenesis. J Clin Invest. 2005;115(8):2083-98.

35. Cui L, Duchamp NS, Boston DJ, et al. NF-KB is involved in brain repair by stem cell factor and granulocyte-colony stimulating factor in chronic stroke. Exp Neurol. 2015;263:17-27.

36. Komine-Kobayashi M, Zhang N, Liu M, et al. Neuroprotective effect of recombinant human granulocyte colony-stimulating factor in transient focal ischemia of mice. J Cereb Blood Flow Metab. 2006:26(3):402-13.

37. Schäbitz W, Kollmar R, Schwaninger M, et al. Neuroprotective effect of granulocyte colony-stimulating factor after focal cerebral ischemia. Stroke. 2003;34(3):745-51.

38. Zhao L-RL, Singhal S, Duan WW-M, et al. Brain repair by hematopoietic growth factors in a rat model of stroke. Stroke. 2007;38(9):2584-91. 
39. Sehara Y, Hayashi T, Deguchi K, et al. Potentiation of neurogenesis and angiogenesis by G-CSF after focal cerebral ischemia in rats. Brain Res. 2007; 1151:142-9.

40. Kawada H, Fujita J, Kinjo K, et al. Nonhematopoietic mesenchymal stem cells can be mobilized and differentiate into cardiomyocytes after myocardial infarction Nonhematopoietic mesenchymal stem cells can be mobilized and differentiate into cardiomyocytes after myocardial infarction. Blood. 2013;104(12):3581-7.

41. Kocher AA, Schuster MD, Szabolcs MJ, et al. Neovascularization of ischemic myocardium by human bone-marrow-derived angioblasts prevents cardiomyocyte apoptosis, reduces remodeling and improves cardiac function. Nat Med. 2001;7(4):430-6.

42. Park H-K, Chu K, Lee S-T, et al. Granulocyte colony-stimulating factor induces sensorimotor recovery in intracerebral hemorrhage. Brain Res. 2005;1041(2):125-31.

43. Diederich $\mathrm{K}$, Quennet $\mathrm{V}$, Bauer $\mathrm{H}$, et al. Successful regeneration after experimental stroke by granulocyte-colony stimulating factor is not further enhanced by constraint-induced movement therapy either in concurrent or in sequential combination therapy. Stroke. 2012;43(1):185-92.

44. Mantovani A, Biswas SK, Galdiero MR, et al. Macrophage plasticity and polarization in tissue repair and remodelling. J Pathol. 2013;229(2):176-85.

45. Gordon S. Alternative activation of macrophages. Nat Rev Immunol. 2003; 3(1):23-35.

46. Mantovani A, Sica A, Sozzani S, et al. The chemokine system in diverse forms of macrophage activation and polarization. Trends Immunol. 2004; 25(12):677-86.

47. Mantovani A, Locati M, Vecchi A, et al. Decoy receptors: a strategy to regulate inflammatory cytokines and chemokines. Trends Immunol. 2001; 22(6):328-36.

48. England TJ, Sprigg N, Alasheev AM, et al. Granulocyte-colony stimulating factor (G-CSF) for stroke: an individual patient data meta-analysis. Sci Rep. 2016. doi:10. 1038/srep36567.

49. Kallmünzer B, Tauchi M, Schlachetzki JC, et al. Granulocyte colonystimulating factor does not promote neurogenesis after experimental intracerebral haemorrhage. Int J Stroke. 2014;9(6):783-8.

50. Diederich K, Schmidt A, Beuker C, et al. Granulocyte colony-stimulating factor (G-CSF) treatment in combination with transplantation of bone marrow cells is not superior to G-CSF treatment alone after cortical stroke in spontaneously hypertensive rats. Front Cell Neurosci. 2014;8:1-9.

51. Ringelstein EB, Thijs $V$, Norrving B, et al. Granulocyte colony-stimulating factor in patients with acute ischemic stroke: results of the AX200 for Ischemic Stroke trial. Stroke. 2013;44(10):2681-7.

52. Schäbitz WR, Laage R, Vogt G, et al. AXIS: a trial of intravenous granulocyte colony-stimulating factor in acute ischemic stroke. Stroke. 2010;41(11):2545-51.

53. Emsley $\mathrm{HC}$ a, Smith CJ, Georgiou RF, et al. A randomised phase II study of interleukin-1 receptor antagonist in acute stroke patients. J Neurol Neurosurg Psychiatry. 2005;76(10):1366-72.

54. Arend WP, Malyak M, Guthridge CJ, et al. Interleukin-1 receptor antagonist: role in biology. Annu Rev Immunol. 1998;16:27-55.

55. Siddappa R, Licht R, Van Blitterswijk C, et al. Donor variation and loss of multipotency during in vitro expansion of human mesenchymal stem cells for bone tissue engineering. J Orthop Res. 2007;25:1029-41.

56. Siegel G, Kluba T, Hermanutz-Klein U, et al. Phenotype, donor age and gender affect function of human bone marrow-derived mesenchymal stromal cells. BMC Med. 2013;11:146.

57. Montzka K, Lassonczyk N, Tschöke B, et al. Neural differentiation potential of human bone marrow-derived mesenchymal stromal cells: misleading marker gene expression. BMC Neurosci. 2009;10:16.

58. Portalska KJ, Groen N, Krenning G, et al. The effect of donor variation and senescence on endothelial differentiation of human mesenchymal stromal cells. Tissue Eng Part A. 2013;19(21-2):2318-29.

59. Huang S, Feng $C$, Wu $Y$, et al. Dissimilar characteristics of umbilical cord mesenchymal stem cells from donors of different ages. Cell Tissue Bank. 2013;14(4):707-13.

60. Ortiz LA, Dutreil M, Fattman C, et al. Interleukin 1 receptor antagonist mediates the antiinflammatory and antifibrotic effect of mesenchymal stem cells during lung injury. Proc Natl Acad Sci U S A. 2007;104(26):11002-7.
61. Capra E, Beretta R, Parazzi V, et al. Changes in the proteomic profile of adipose tissue-derived mesenchymal stem cells during passages. Proteome Sci. 2012;10(1):46.

62. Czekanska EM, Ralphs JR, Alini M, et al. Enhancing inflammatory and chemotactic signals to regulate bone regeneration. Eur Cell Mater. 2014;28: 320-34.

\section{Submit your next manuscript to BioMed Central and we will help you at every step:}

- We accept pre-submission inquiries

- Our selector tool helps you to find the most relevant journal

- We provide round the clock customer support

- Convenient online submission

- Thorough peer review

- Inclusion in PubMed and all major indexing services

- Maximum visibility for your research

Submit your manuscript at www.biomedcentral.com/submit

) Biomed Central 